\title{
Comment survivre dans l'ombre d'un géant : le cas des plateformes de covoiturage de petite et moyenne taille en Chine
}

\author{
Shouheng Sun ${ }^{\mathrm{a}}$
}

RÉSUMÉ. Cet article vise à explorer comment les plateformes de l'économie collaborative de petite et moyenne taille obtiennent un avantage concurrentiel et ont la chance de survivre et de se développer sur un marché monopolisé par des géants de l'industrie. Nous prenons l'exemple du marché chinois du covoiturage, pour lequel une étude de cas a été menée en utilisant la matrice de modèle d'affaires comme outil d'analyse. Trois catégories typiques de plateformes ont été résumées à partir de 128 plateformes de mobilité intelligente de transport de petite et moyenne taille. En comparant et en analysant la matrice de modèle d'affaires entre ces trois types de plateformes et Didi ${ }^{1}$, le géant chinois de l'industrie technologique, on constate que les plateformes de covoiturage de petite et moyenne taille peuvent développer un avantage concurrentiel unique en offrant une proposition de valeur différenciée et en construisant la matrice de modèle d'affaires correspondant dans un domaine spécifique. Afin de gagner plus d'espace sur le marché, ils devraient constamment effectuer une segmentation du marché et une identification des utilisateurs plus sophistiquées dans le but de rechercher de nouvelles occasions commerciales.

\begin{abstract}
This paper aims to explore how do the small and medium-sized collaborative economy platforms gain competitive advantage and get the chance to survive and develop in a market monopolized by industry giants. We take the ride-hailing market in China as an example, for which a case study was conducted by utilizing the business model canvas as an analysis tool. Three typical categories of platforms were summarized from 128 small and medium-sized ride-hailing platforms. By comparing and analyzing the business model canvas between these three types of platforms and the Chinese industry giant Didi, we find that small and medium-sized ride-hailing platforms can develop unique competitive advantage by proposing differentiated value proposition and by constructing corresponding business model canvas in a specific field. In order to gain more market space, they should constantly carry out more sophisticated market segmentation and user identification to look for new business opportunities.
\end{abstract}

\section{Introduction}

Les récents développements en technologie numérique ont donné aux consommateurs plus de pouvoir que jamais, favorisant ainsi l'émergence d'une reconfiguration importante de l'échange marchand sous différentes formes (Ertz, Hallegatte et Bousquet, 2019). Une des plus marquantes parmi celles-ci est l'économie collaborative, qui a apporté des changements révolutionnaires à la conceptualisation de l'échange marchand. L'économie collaborative a aussi été désignée sous le nom d'« économie du partage », qui se définit comme étant un modèle socioéconomique reposant sur l'utilisation partagée de produits sous-utilisés ou indésirables (Botsman et Rogers, 2010). Elle est définie de manière générale par des échanges entre pairs au moyen de plateformes Internet (p. ex., plateformes d'économie collaborative) qui facilitent les échanges ou la location de ressources inutilisées, comme des produits matériels, de l'espace, des ressources et de la main-d'œuvre, et ce, en temps réel (Sundararajan, 2014).

a Postdoctorant en marketing, LaboNFC, Université du Québec à Chicoutimi 
L'économie collaborative a connu une croissance exponentielle au cours de la dernière décennie. Ainsi, une multitude de plateformes d'économie collaborative (PEC) se sont rapidement développées et ont réalisé des progrès notables. Cette nouvelle reconfiguration de l'échange marchand, qui offre l'accès à des commodités pratiques à faible coût, mais sans responsabilités liées à la possession de ces dernières (Eckhardt et Bardhi, 2015) a perturbé divers domaines bien établis et a modifié de manière considérable toutes les facettes de la vie des gens. Elle s'est répandue dans plusieurs secteurs, par exemple les produits partagés (voitures, équipements, jouets, vêtements, etc.), les espaces partagés (habitation, places de stationnement, terrain, etc.), le partage de connaissances et de compétences (connaissances, expériences, etc.), la main-d'œuvre partagée (entretien ménager, buanderie, etc.), les finances partagées (financement participatif de produit, en actions, etc.) et la capacité de production partagée (énergie, infrastructure informatique) (European Commission, 2016; Hamari, Sjöklint et Ukkonen, 2016; Smith, 2016).

L'essor de l'économie collaborative a suscité beaucoup d'intérêt dans les milieux universitaires (Frenken et Schor, 2017; Ertz et Leblanc-Proulx, 2018). Les chercheurs ont mené des études étendues et approfondies sur l'économie collaborative sous plusieurs angles. Par exemple, certains ont examiné les innovations du modèle d'affaires des chefs de file, comme Uber et Airbnb, et ont analysé les répercussions négatives de leur expansion rapide sur l'industrie traditionnelle de leur secteur (Mikhalkina et Cabantous, 2015; Bashir, Yousaf et Verma, 2016,). De plus, certains chercheurs se sont intéressés aux enjeux de gouvernance soulevés par le développement des PEC (Edelman et Geradin, 2015). Un des plus importants est le monopole de l'industrie pouvant donner lieu à des problèmes reliés à l'opacité de la tarification des géants d'industrie, par exemple Uber (Gabel, 2016). En outre, certaines problématiques sociales telles que la discrimination, le droit du travail et la confiance envers l'économie collaborative sont également des sujets d'actualité pour le milieu universitaire (Ert, Fleischer et Magen, 2016; Frenken et Schor, 2017). Par exemple, des études ont démontré qu'il existe un certain degré de discrimination raciale dans le processus transactionnel des plateformes d'Airbnb et d'Uber (Edelman, Luca et Svirsky, 2017; Hua et Ray, 2018).
Néanmoins, presque toutes les études sur l'économie collaborative se concentrent principalement sur les géants en position de monopole, à savoir Uber et Airbnb, qui possèdent la majorité des parts du marché et qui sont présentes de manière privilégiée dans les grands centres urbains. Jusqu'à présent, peu d'information est disponible sur les nombreuses PEC de petite et moyenne taille sur le marché, bien qu'elles soient également une partie très importante de l'économie collaborative. On ignore de quelle manière ces PEC de petite et moyenne taille contribuent à la reconfiguration de l'échange marchand et amènent des avancées conduisant à cette reconfiguration. Notamment, deux questions très importantes méritent des recherches approfondies : De quelle façon obtiennent-elles un avantage concurrentiel? Comment survivent-elles et se développent-elles dans un marché monopolisé? Plus spécifiquement, il est question de savoir quelles sont les caractéristiques principales qui les différencient de ces géants d'industrie, par exemple leurs clients, la valeur apportée à leurs clients, la façon dont elles font des profits, etc. Cela constituera la matrice de modèle d'affaires qui pourra répondre systématiquement aux questions précédentes. Cette matrice de modèle d'affaires est le scénario et le schéma directeur qui illustrent de quelle façon les entreprises exercent leurs activités commerciales et qui démontrent que les entreprises peuvent prospérer simplement grâce à une matrice de modèle d'affaires bien conçue.

Par conséquent, afin de combler cette lacune en matière de recherche, cet article prend comme exemple le marché du covoiturage en Chine et effectue une étude de cas en se servant de la matrice de modèle d'affaires comme outil d'analyse. En comparant et en analysant les matrices de modèle d'affaires des géants de l'industrie et des plateformes de petite et moyenne taille, cette recherche tente de présenter un portrait clair reflétant les caractéristiques et les initiatives des PEC de petite et moyenne taille, plus précisément dans le marché du covoiturage en Chine, et de jeter un regard nouveau sur la reconfiguration de l'échange marchand selon la perspective de ces entreprises de petite et moyenne taille. 


\section{Méthodologie et outils}

Ces dernières années, la matrice de modèle d'affaires a largement suscité l'intérêt des chercheurs et des professionnels (Cosenz, 2017) en raison de son précieux rôle en simulation, en analyse, en compréhension et en développement des concepts d'affaires présents ou nouveaux. De manière générale, on la définit comme étant l'architecture de l'économie et de la structure organisationnelle d'une entreprise (Amit et Zott, 2015). Dans la documentation récente sur la modélisation d'entreprises, plusieurs archétypes ont été examinés et recommandés (Baden-Fuller et Mangematin, 2013; Fjeldstad et
Snow, 2018; Teece, 2010). Parmi ceux-ci, la «matrice de modèle d'affaires à trois niveaux » (MMA) semble être la plus efficace en communication de stratégie d'affaires (Joyce et Paquin, 2016). En effet, dans le but de mettre en place une méthode normalisée pour la conception de matrices de modèle d'affaires, Osterwalder et Pigneur (2010) ont conçu un cadre qui permet de mieux illustrer une nouvelle stratégie d'affaires et son organisation, et cette information peut être facilement partagée grâce à une conception allégée et pertinente. Ce cadre spécifique de la MMA est illustré à la figure 1.

\begin{tabular}{|l|l|l|l|l|}
\hline \multirow{2}{*}{$\begin{array}{l}\text { Partenaires } \\
\text { essentiels }\end{array}$} & $\begin{array}{l}\text { Activités } \\
\text { essentielles }\end{array}$ & Proposition de & $\begin{array}{l}\text { Relation avec la } \\
\text { clientèle }\end{array}$ & \\
\cline { 2 - 2 } & $\begin{array}{l}\text { Ressources } \\
\text { essentielles }\end{array}$ & & $\begin{array}{l}\text { Segment de } \\
\text { clients }\end{array}$ \\
\cline { 4 - 5 } & & Canaux & \\
\hline Structure des coûts & & \multicolumn{2}{|l}{} \\
\hline
\end{tabular}

Figure 1 - Cadre de la matrice de modèle d'affaires Source : Osterwalder et Pigneur, 2010

Comme le montre la figure 1, la matrice de modèle d'affaires (MMA) est structurée selon neuf éléments constitutifs en lien avec les principaux facteurs qui représentent la dynamique d'affaires sous-jacente au processus de création de valeur économique :

1) Partenaires essentiels: Incluent les principaux intervenants impliqués dans le processus de création de valeur, plus particulièrement ceux qui font que l'entreprise fonctionne (p. ex., fournisseurs, donneurs de subventions, bailleurs et autres);

2) Activités essentielles: Décrivent les activités centrales et les processus générant de la valeur (p. ex., production, fabrication);

3) Ressources essentielles: Par exemple, les actifs matériels et immatériels liés aux facteurs critiques de succès qui doivent être achetés et accumulés, qui font partie de la routine organisationnelle et qui seront déployés ultérieurement pour contribuer au processus de création de valeur;

4) Proposition de valeur: Par exemple, l'adéquation entre les besoins des clients et la valeur créée par l'entreprise afin de les satisfaire;
5) Relation avec la clientèle: Définit de quelle façon l'entreprise interagit avec ses clients et quelles sont ses méthodes de fidélisation de la clientèle;

6) Canaux: Illustrent comment la distribution de produits et services est effectuée;

7) Segment de clients : Par exemple, composition de la clientèle (personnes ou entreprises);

8) Structure des coûts: Décrit les coûts liés au fonctionnement de l'entreprise en mettant l'accent sur l'acquisition de ressources; et

9) Sources de revenus: Définissent les prix des produits et services ainsi que le volume prospectif des ventes.

La MMA présente dans un cadre unique non seulement les forces internes reliées à l'organisation et au fonctionnement de l'entreprise, mais aussi quelques sources externes qui affectent l'entreprise et qui sont affectées par les activités de l'entreprise (p. ex., clients et partenaires). La perspective qu'apporte la MMA permet aux intervenants de comprendre rapidement de quelle façon l'entreprise fonctionne, sa 
valeur potentielle en matière de profitabilité et sa pertinence face aux besoins des clients. Par conséquent, la lisibilité de la MMA et sa capacité à communiquer facilement l'information sur le fonctionnement d'une entreprise sont ses principales forces (Cosenz, 2017). C'est pourquoi cet article utilise la MMA en tant que cadre théorique pour une analyse empirique.

La plateforme de covoiturage, agissant comme un lien entre l'offre et la demande, permet de créer rapidement des correspondances entre passagers et conducteurs, et de conclure la transaction grâce à la mise en place d'une série de mécanismes tels que les applications mobiles de services basés sur la location (SBL), les algorithmes et tarifs dynamiques ainsi qu'un système d'évaluation mutuelle pour les deux parties (Clewlow et Mishra, 2017). L'essor de l'économie collaborative a stimulé le développement rapide des plateformes de covoiturage à travers le monde. Certaines d'entre elles ont obtenu des résultats remarquables, dont Uber.

Inspirées par le succès d'Uber, des centaines de plateformes de covoiturage ont successivement vu le jour en Chine depuis 2010 (China State Information Center, 2018). Avec le soutien du capital de risque, l'ensemble de l'industrie des services de covoiturage est entré dans un contexte extrêmement concurrentiel durant plusieurs années. La majorité de ces entreprises de plateformes ont investi des milliards de dollars dans une guerre de « combustion de capital» à grande échelle pour s'approprier des parts de marché. En 2016, avec la fusion d'Uber Chine et de Didi, cette dernière est devenue la plus grande plateforme de covoiturage au pays. À l'heure actuelle, il existe encore des centaines d'entreprises de covoiturage en ligne en Chine. Parmi celles-ci, Didi occupe environ $90 \%$ du marché (Finance, 2018). Les autres plateformes de covoiturage sont toutes des entreprises de petite et moyenne taille.

Grâce à des recherches sur Internet, à des enquêtes pratiques et à des analyses statistiques basées sur des rapports d'industries connexes et sur un annuaire statistique régional (Analysys International, 2019; National Bureau of Statistics of China, 2019; China Industry Research Institute, 2018; China State Information Center, 2019), nous avons recueilli et trié les informations de 128 entreprises de plateformes de covoiturage de petite et moyenne taille fondées en Chine entre 2016 et 2017. Cela couvre presque tous les types d'entreprises de plateformes de covoiturage de petite et moyenne taille en Chine, et peut être divisé selon les catégories suivantes :

1) Catégorie 1: Les plateformes de petite et moyenne taille ayant le même modèle de fonctionnement que Didi et qui concurrencent Didi sur tous les plans dans les villes;

2) Catégorie $2:$ Les plateformes qui sont passées d'entreprise traditionnelle de location de voitures à fournisseur de services de covoiturage de bas et de milieu de gamme;

3) Catégorie 3: Les plateformes de covoiturage haut de gamme (p. ex., HBP), conçues pour la clientèle des entreprises haut de gamme dans les villes de troisième rang et de deuxième rang;

4) Catégorie 4 : Les plateformes en ligne de covoiturage par minibus (p. ex., CPM), qui offrent des trajets domicile-travail pour les employés de bureau ordinaires qui vivent en banlieue, mais qui travaillent au centre-ville;

5) Catégorie 5: Les plateformes de covoiturage interurbain, qui fournissent des services de voyage intervilles flexibles et pratiques pour les gens qui ont besoin d'effectuer des déplacements interurbains.

Puis, nous résumons et analysons les modes de fonctionnement de ces 128 plateformes et leur processus de développement à la fin de 2019. Durant cette période de recherche, Didi n'a cessé de développer ses avantages concurrentiels. En raison de la concurrence féroce du marché, les plateformes de la catégorie 1 ont obtenu de mauvais résultats en matière de taille et de rentabilité, et la plupart se sont retirées du marché. De même, la majorité des plateformes de la catégorie 2 ont éventuellement renoncé à fonctionner de manière indépendante et ont établi un partenariat avec Didi en tant que fournisseur de véhicules. Toutefois, le développement des trois autres types de plateformes (catégories 3 à 5) est relativement bon, et la taille de la flotte et le nombre d'utilisateurs sont en constante augmentation. Ainsi, nous avons sélectionné en tant qu'exemples ces trois types de plateformes de petite et moyenne taille, puis nous avons comparé et analysé les différences entre leurs modèles commerciaux et ceux des géants de l'industrie à l'aide de la MMA comme outil d'analyse. 


\section{Résultats}

\subsection{Matrice de modèle d'affaires de Didi}

En nous basant sur des études antérieures (Kim, Baek et Lee, 2018; Täuscher et Laudien, 2018), puis en les combinant avec les antécédents de croissance et les conditions d'exploitation actuelles de Didi, nous résumons brièvement la matrice de modèle d'affaires de Didi et nous la comparons au cadre de la MMA équivalent. Les résultats explicites sont présentés à la figure 2 .

\begin{tabular}{|c|c|c|c|c|}
\hline $\begin{array}{l}\text { Investisseurs/ } \\
\text { spécialistes en } \\
\text { capital de risque } \\
\text { Fournisseurs de } \\
\text { technologie et } \\
\text { d'infrastructure } \\
\text { (ex. : navigation } \\
\text { sur carte, } \\
\text { plateformes de } \\
\text { paiement tierce } \\
\text { partie) }\end{array}$ & $\begin{array}{c}\text { ACTIVITÉS } \\
\text { ESSENTIELLES } \\
\text { Augmenter la taille } \\
\text { de l'offre et de la } \\
\text { demande } \\
>\text { Réduire les facteurs } \\
\text { externes négatifs } \\
>\text { Augmenter les } \\
\text { avancées } \\
\text { technologiques et la } \\
\text { propriété } \\
\text { intellectuelle } \\
>\text { Améliorer la } \\
\text { proposition de } \\
\text { valeur } \\
>\text { Élargir le marché et } \\
\text { le champ d'activité }\end{array}$ & $\begin{aligned} & \text { PROPOSITION } \\
& \text { DE VALEUR } \\
& \text { Passagers } \\
&>\quad \text { Prix plus bas que } \\
& \text { les taxis normaux } \\
&>\quad \text { Ramassages } \\
& \text { rapides } \\
&>\text { Pratique } \\
&>\text { Sûr et sécuritaire } \\
& \text { Conducteurs } \\
&>\quad \text { Occasion de } \\
& \text { gagner de l'argent } \\
&>\text { Horaires de } \\
& \text { travail flexibles }\end{aligned}$ & 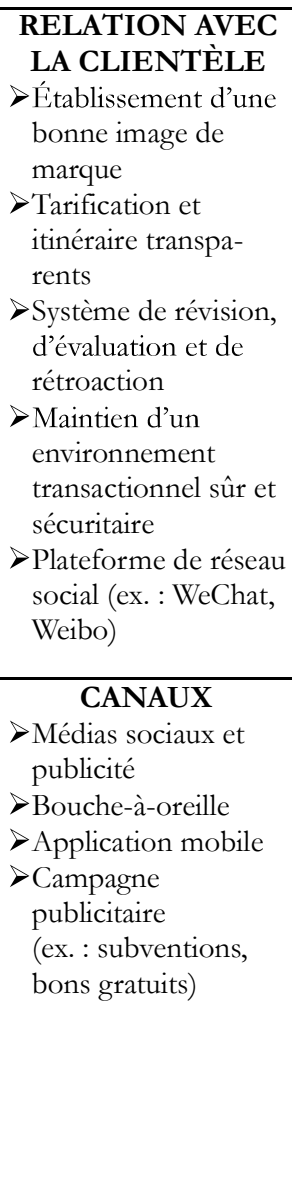 & 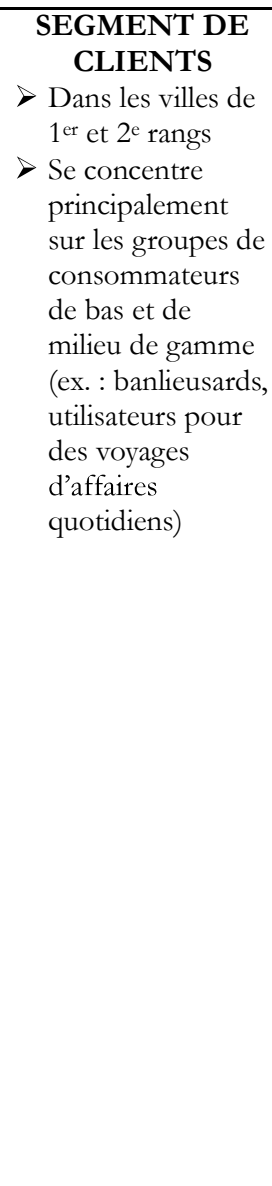 \\
\hline \multicolumn{2}{|c|}{$\begin{array}{l}\text { STRUCTURE DES COÛTS } \\
\text { Infrastructure technologique et espace de } \\
\text { travail } \\
>\text { Coûts de gestion du fonctionnement de la } \\
\text { plateforme (ex. : coûts des ressources } \\
\text { humaines) } \\
>\text { Publicité et autres investissements } \\
\text { d'entreprise } \\
>\text { Investissements en technologie de R-D }\end{array}$} & \multicolumn{3}{|c|}{$\begin{array}{l}\text { SOURCES DE REVENUS } \\
>\text { Commissions des services de covoiturage } \\
>\text { Recettes publicitaires provenant de compagnies tierces (affichage de } \\
\text { publicité dans les voitures et sur l'application mobile) } \\
>\text { Gestion de capital } \\
>\text { Autres revenus de coopération entre entreprises }\end{array}$} \\
\hline
\end{tabular}

Figure 2 - Matrice de modèle d'affaires de Didi 


\section{Partenaires essentiels}

Didi a trois importants types de partenaires essentiels. Le premier concerne les véhicules et les conducteurs, qui sont les composantes les plus importantes de l'offre de la plateforme de covoiturage et qui servent à remplir la proposition de valeur aux clients finaux. Les conducteurs possédant leur propre véhicule sont la composante principale de l'offre de la plateforme. Le deuxième type est les partenaires technologiques. Ils simplifient l'accompagnement de l'offre et de la demande en aidant à effectuer des transactions efficaces, ce qui permet à la plateforme de réaliser sa proposition de valeur unique. Ces partenaires technologiques comprennent des systèmes de navigation sur carte, des plateformes de paiement tierce partie, des services d'utilisation de terminaux mobiles, etc. Enfin, les investisseurs et les spécialistes en capital de risque constituent le plus puissant soutien et la source de financement la plus importante durant la croissance de la plateforme, particulièrement lors de la phase de démarrage du développement.

\section{Activités essentielles}

Quant aux activités essentielles, l'impact de la taille est l'ingrédient essentiel des entreprises de plateformes pour obtenir l'avantage concurrentiel. Par conséquent, les activités essentielles devraient continuellement augmenter la taille de l'offre et de la demande pour la plateforme. Parallèlement, la plateforme devrait constamment diminuer les facteurs négatifs externes afin de créer un environnement externe favorable, dont le soutien gouvernemental et l'approbation du public. De plus, la plateforme devrait continuer d'améliorer sa proposition de valeur et de rechercher des propositions de valeur complémentaires pour mieux répondre aux besoins des clients et ainsi attirer plus de participants à se joindre à la plateforme. Enfin et surtout, la plateforme devrait augmenter les avancées technologiques et la propriété intellectuelle pour accentuer les obstacles à l'entrée du marché ainsi que pour également étendre le marché (p. ex., s'étendre à plus de villes) et le champ d'activité (p. ex., livraison de nourriture, logistique, services de voyage et autres entreprises) afin de chercher de nouveaux espaces de développement et un nouveau stade de croissance des bénéfices.

\section{Ressources essentielles}

La ressource principale de la plateforme est l'impact de la taille, qui doit être construite et alimentée.
À l'ère des mégadonnées, les données, les algorithmes et la capacité d'analyse ainsi que les renseignements sur les gains sont essentiels pour les entreprises, particulièrement pour les plateformes numériques. Avec l'expansion continue de la taille des plateformes, un grand nombre de données et d'informations sur les usagers, sur les conducteurs et sur les véhicules seront générées et accumulées, ce qui deviendra la richesse la plus précieuse de la plateforme. De plus, la capacité de la plateforme à innover et à se développer est aussi très importante, car elle peut la soutenir dans l'expansion continue du champ d'activité et des innovations du modèle d'affaires pour parvenir à une croissance soutenue. L'importance de la marque est l'un des actifs immatériels les plus importants d'une entreprise; elle est également une ressource essentielle dans le processus de développement d'une plateforme.

\section{Proposition de valeur}

La plateforme de covoiturage est une plateforme multifacette. De ce fait, elle doit offrir une proposition de valeur pour les deux parties : les passagers autant que les conducteurs. Pour les passagers, la proposition de valeur consiste à répondre à la demande des clients en matière de déplacement en voiture (p. ex., offrir des services personnalisés et diversifiés, à prix modique, pratiques, avec une garantie de ramassage rapide et sécuritaire). Pour les conducteurs, c'est l'occasion de gagner un revenu supplémentaire et la liberté de choisir ses heures de travail.

\section{Relation avec la clientèle}

Elle comprend l'établissement d'une bonne image de marque, l'offre de prix et d'itinéraires transparents, le maintien d'un environnement transactionnel sûr et sécuritaire, la mise en place d'un système de révision, d'évaluation et de rétroaction ainsi que l'accroissement des interactions par le biais d'une plateforme de réseau social (p. ex., WeChat, Weibo).

\section{Canaux}

Les canaux de Didi pour se faire connaître et pour développer son marché sont généralement des campagnes publicitaires (p. ex., subventions, bons gratuits), les médias sociaux et la publicité, le bouche-à-oreille et l'application mobile. 


\section{Segment de clients}

Voici certaines des segmentations les plus importantes et utiles : l'aspect démographique (p. ex., âge, situation socioéconomique, situation familiale); l'aspect géographique, qui représente le secteur du marché (p. ex., grande ou petite ville, banlieue); le comportement des consommateurs et les habitudes de consommation (p. ex., préférence de prix ou de qualité du service, fréquence d'utilisation). Considérant le phénomène d'agglomération et l'importance de la consommation, le principal marché de Didi se situe dans les villes de premier et deuxième rangs, et se concentre principalement sur les groupes de consommateurs de moyenne gamme et de bas de gamme (p. ex., banlieusards, utilisateurs pour des voyages d'affaires quotidiens).

\section{Structure des coûts}

Pour Didi, le principal facteur de coûts des premiers stades de développement est le coût d'acquisition des usagers et des conducteurs, qui est "subventionné » par Didi. De plus, il existe aussi les principaux types de dépenses liés au fonctionnement, dont l'infrastructure technologique et l'espace de travail, les coûts de gestion du fonctionnement de la plateforme (p. ex., coûts des ressources humaines), la publicité et les autres investissements d'entreprise, ainsi que des investissements en technologie de recherche-développement.

\section{Source de revenus}

Elles regroupent principalement les commissions des services de covoiturage, les recettes publicitaires provenant de compagnies tierces (affichage de publicité dans les voitures et sur l'application mobile), la gestion de capital et les autres revenus de coopération entre entreprises.

\subsection{Plateformes haut de gamme de covoiturage commercial}

Puisque le prix d'un service de covoiturage en ligne est généralement plus bas de $10 \%$ à $25 \%$ que celui des taxis urbains normaux, il a grandement stimulé la demande des consommateurs grand public, particulièrement des groupes de consommateurs de moyenne gamme et de bas de gamme, ce qui a ainsi permis à la plateforme de se construire rapidement une base d'utilisateurs. Didi a connu une croissance rapide en ciblant les consommateurs de masse qui recherchent des services abordables et pratiques.

Contrairement à Didi, HBP est destinée à une clientèle d'affaires haut de gamme dans les villes de premier et deuxième rangs. Sa clientèle cible est constituée des personnes qui veulent une expérience de qualité dans leur vie quotidienne, telles que les gens d'affaires ayant une réunion importante ou encore les familles qui profitent de leur fin de semaine ou qui organisent une célébration spéciale.

Nous utilisons le prix d'un taxi urbain normal comme valeur de référence et observons l'impact des changements de tarifs pour les utilisateurs. Le résultat de l'analyse de la sensibilité à la tarification des utilisateurs du marché du covoiturage en Chine est présenté à la figure 3 .

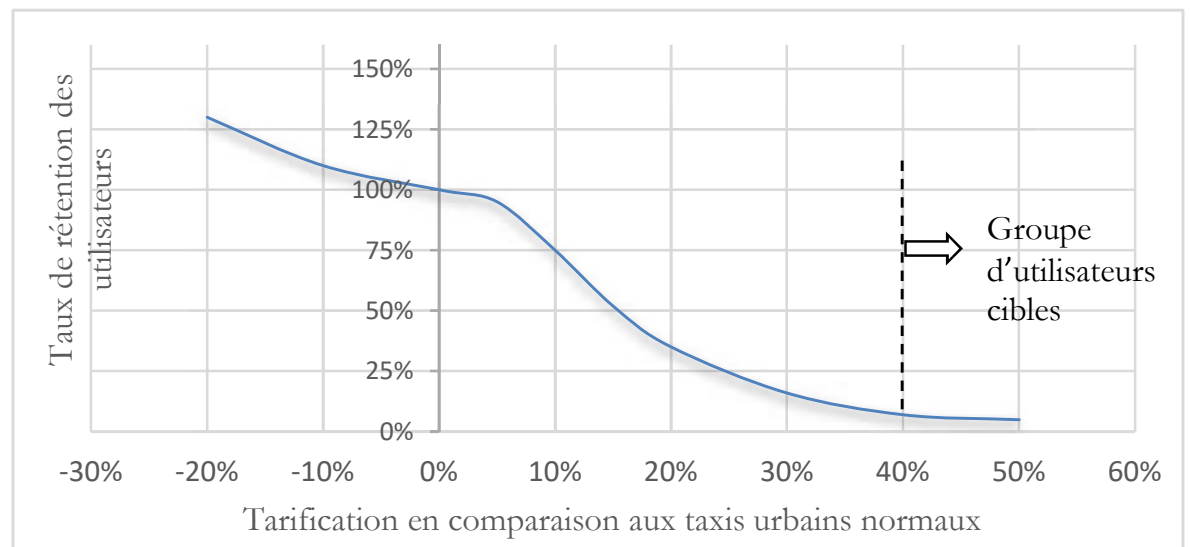

Note : Ces statistiques sont basées sur Analysys International (2019), China State Information Center (2019) et Berger (2016)

Figure 3 - Analyse de la sensibilité à la tarification des utilisateurs du marché du covoiturage en Chine 
Avec l'augmentation progressive du tarif, le nombre d'utilisateurs a graduellement diminué. Le groupe d'utilisateurs cibles d'HBP est les gens capables d'accepter un tarif augmenté de plus de $40 \%$, dépassant même les $100 \%$. Ces plateformes adoptent un modèle de gestion de conducteurs et de véhicules différent de celui de Didi : elles ont leur propre flotte et ont recruté des conducteurs à temps plein. Les conducteurs sont embauchés après une sélection rigoureuse et après avoir suivi une formation professionnelle. Les normes de service et de sécurité sont nettement supérieures aux plateformes de covoiturage ordinaires. En comparaison avec la plateforme de Didi, celle d'HBP possède les avantages suivants :

1) Elle a une base d'utilisateurs plus stable, alors que la base d'utilisateurs de Didi est sensible à la tarification. Ce petit groupe d'utilisateurs a une sensibilité à la tarification très basse, accorde plus d'importance à la qualité du service et a une loyauté plus élevée;

2) Les risques de sécurité, tels que les compétences de conduite et les informations criminelles, ne peuvent pas être entièrement contrôlés et sont devenus des facteurs d'incertitude pour le marché chinois de covoiturage en ligne. Par exemple, Didi a subi récemment les effets d'une série d'évènements négatifs, tels que des passagers battus, du harcèlement sexuel et des meurtres. La mise à disposition de conducteurs à temps plein permet d'améliorer grandement la sécurité du service, particulièrement pour ceux qui souhaitent obtenir des déplacements sécuritaires et de haute qualité;

3) Certaines de ces plateformes sont des marques dérivées créées par des fabricants automobiles célèbres, par exemple ReachNow avec le soutien de BMW (Baijiahao, 2018) et Shouqi avec le soutien de Volkswagen (Shouqi, 2020). Les autres sont des entreprises professionnelles de location de voitures qui existent depuis plusieurs années, par exemple Ucar et Yongche. Elles ont des avantages naturels en matière de coût des véhicules et d'importance de la marque dans ce segment du marché.

Actuellement, les services de covoiturage commerciaux haut de gamme ne sont que l'ébauche d'un marché supplémentaire en Chine. Avec l'amélioration continue du niveau socioéconomique et de la capacité de consommation, ce type de plateforme gagnera en possibilités de développement élargies.

\subsection{Plateformes de covoiturage par minibus}

En raison du grave déséquilibre entre l'offre et la demande des transports en commun dans plusieurs grandes villes chinoises, la demande du marché pour le covoiturage en ligne est toujours énorme. Par exemple, à Beijing, le ratio entre le nombre d'usagers du transport en commun et le nombre de sièges disponibles pour le transport en commun est de 100:5 (Beijing Bureau of Statistics, 2019; Beijing Transportation Development Research Institute, 2019). La majorité des navetteurs voyagent dans des autobus et métros bondés, et l'expérience utilisateur est très mauvaise (Baidu, 2019). Les politiques de restriction d'achat d'automobile dans plusieurs grandes villes font également en sorte que plus de gens ayant besoin d'une voiture privée ne peuvent se procurer un véhicule. Par conséquent, ils choisissent le covoiturage en ligne comme seul moyen de transport. Néanmoins, en raison de la restriction du nombre de véhicules et de l'espace urbain, la capacité de transport d'une plateforme normale de covoiturage (p. ex., Didi) est très limitée, particulièrement durant l'heure de pointe $\mathrm{du}$ matin et du soir. C'est ainsi que les plateformes de covoiturage par minibus (p. ex., CPM) sont apparues.

Leurs principaux groupes d'utilisateurs sont les employés de bureau qui vivent dans les banlieues, mais qui travaillent au centre-ville. Ils utilisent l'application mobile pour réserver en ligne, pour choisir leur siège, pour payer et pour d'autres opérations (Rainbow Bus, 2020). Les itinéraires et modes de fonctionnement sont semblables aux autobus publics. Toutefois, ils effectuent moins d'arrêts, ce qui permet de ne faire aucun arrêt entre la maison et le bureau. Le nombre de sièges dans les minibus est normalement de 9 à 15, tandis que le nombre moyen de passagers par véhicule de Didi est d'environ 2 (Didi, 2016). Le tarif est également plus bas que celui de Didi. Ainsi, les services de CPM peuvent mieux répondre à la demande des utilisateurs et réduire la pression de la congestion routière dans une large mesure, particulièrement durant les heures de pointe. Aussi, les services d'une plateforme telle que CPM sont plus confortables que les autobus publics et plus sécuritaires que plusieurs plateformes de covoiturage par minibus, qui contiennent plus de passagers par voiture. Au cours 
des deux dernières années, plusieurs de ces plateformes telles que Rainbow Bus, Smooth Home Bus et Shuttle Bus se sont développées rapidement. Elles sont axées sur les itinéraires qui vont des banlieues à forte densité vers le centre-ville, offrant des services de déplacement pour travailleurs.

En comparaison avec Didi, les véhicules appartiennent à la plateforme. Aussi, les véhicules et les conducteurs sont sous son contrôle; elle peut ainsi offrir un volume de services stable. Aussi, puisqu'actuellement la Chine promeut vigoureusement les véhicules à énergies nouvelles (p. ex., voitures électriques), la plupart des plateformes adoptent des véhicules à énergies nouvelles, ce qui offre de bonnes conditions externes pour le développement de la plateforme. D'un autre côté, la plateforme a reçu le soutien des politiques gouvernementales et des subventions de capital. D'autre part, les fabricants de véhicules à énergies nouvelles participent aussi activement à travers diverses formes de collaboration, dont le développement conjoint, qui a grandement réduit les coûts d'exploitation de la plateforme. Ainsi, il y aura plus de place pour un développement au sens large des plateformes de covoiturage par minibus.

\subsection{Plateformes de covoiturage interurbain}

Le service de covoiturage en zone urbaine est relativement au point. Il est même devenu une partie intégrante de la vie des citoyens chinois, particulièrement dans les villes de premier et deuxième rangs. Toutefois, le déplacement entre la ville et les zones rurales, ainsi qu'entre les villes de troisième, quatrième et cinquième rangs, repose encore essentiellement sur des modes de transport traditionnels, tels que les autocars et les trains. En raison du développement inégal des villes chinoises, la répartition des ressources en transport est elle aussi inégale (National Bureau of Statistics of China, 2019). La capacité optimale de transport des villes de quatrième et cinquième rangs est difficile à atteindre à cause des besoins croissants de déplacement diversifié des gens. Plus spécialement en raison de la forte population, lorsqu'arrivent les congés, notamment la Fête du printemps, le transport devient un problème complexe. Avec le développement rapide du covoiturage dans les grandes villes, l'émergence des plateformes de covoiturage interurbain offre une nouvelle façon d'atténuer ce problème. La Chine s'efforce de promouvoir l'urbanisation et l'intégration des zones urbaines et rurales, et les embouteillages entre les villes sont de plus en plus fréquents. La gamme de services des plateformes de covoiturage interurbain est présentée à la figure 4 .
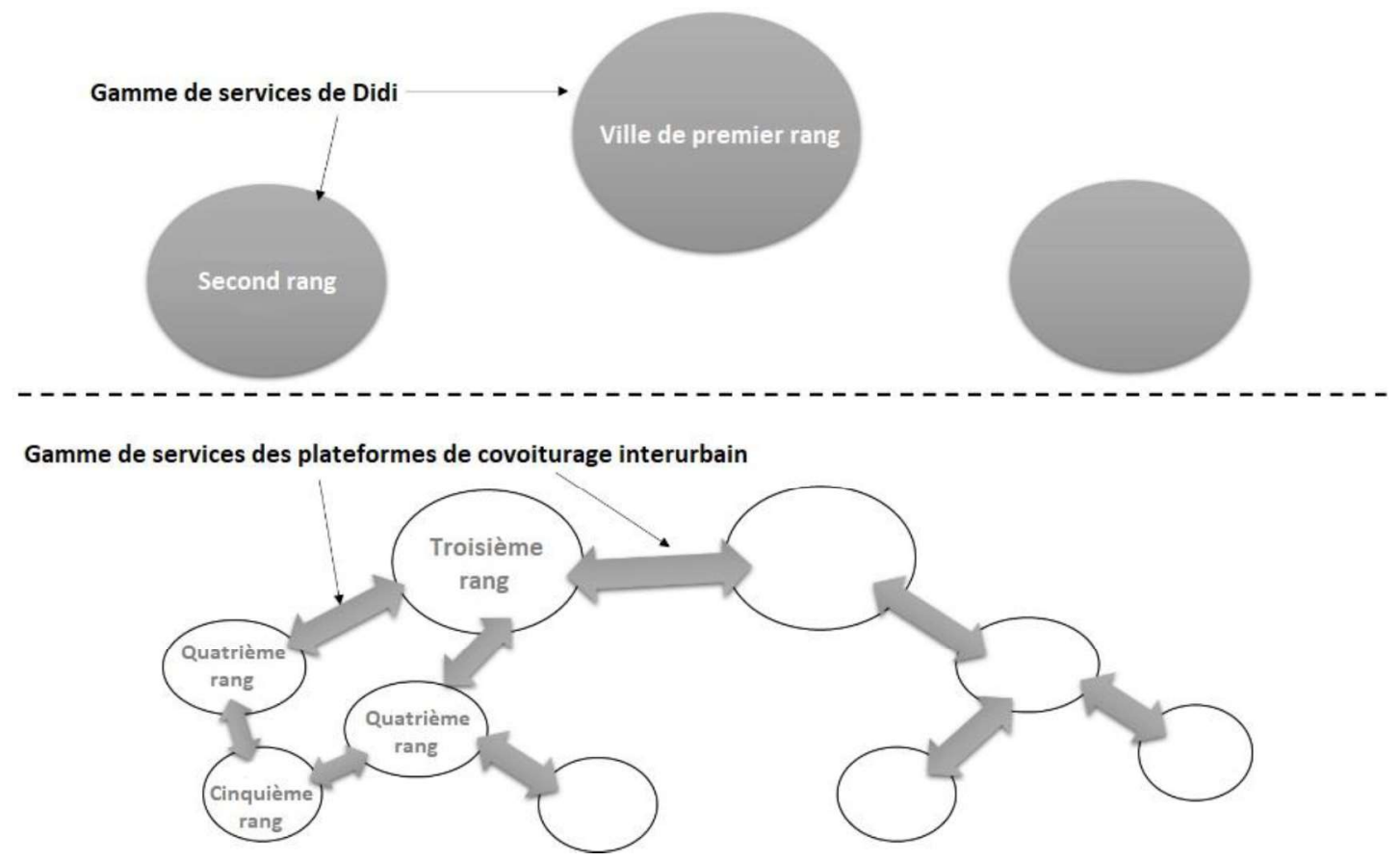

Figure 4-Gamme de services des plateformes de covoiturage interurbain. 
Les caractéristiques principales des plateformes de covoiturage interurbain sont les suivantes :

1) Service de navette porte à porte : Dans certains secteurs, elles peuvent offrir des services d'embarquement et de débarquement point à point ou porte à porte, ce qui réduit ainsi les désagréments de l'attente et des correspondances pour les passagers. Notamment, ce service réduit les tracas des passagers à utiliser les autobus, métros ou taxis en les transportant directement de la maison à leur destination;

2) Coût du vébicule relativement bas: Il est sensiblement le même que le tarif d'un autocar interurbain traditionnel et réduit les frais autrefois liés au changement de moyen de transport;

\section{3) Horaires d'exploitation plus flexibles.}

Ces plateformes de petite taille présentent de fortes caractéristiques régionales. Elles fonctionnent de manière très flexible et ont une bonne base d'utilisateurs dans certains secteurs. Il existe plus de 300 villes de troisième, quatrième et cinquième rangs en Chine, pour une population d'environ 500 millions (National Bureau of Statistics of China, 2019). Même si les marchés sont plus petits et plus fragmentés, suivant le progrès rapide du processus d'urbanisation du pays, les différents besoins variés en transport des gens augmenteront progressivement, ce qui créera sans aucun doute beaucoup plus de place pour le développement de telles plateformes.

\section{Discussion}

\section{Comparaison des trois types de plateformes avec la $M M A$ de Didi}

Au moyen de l'analyse des trois types de plateformes, nous avons résumé certaines des différentes composantes significatives des matrices de modèle d'affaires en les comparant à celles de Didi (voir tableau 1).

\begin{tabular}{|c|c|c|c|c|}
\hline Composante & Didi & $\begin{array}{c}\text { Plateformes haut } \\
\text { de gamme de } \\
\text { covoiturage } \\
\text { commercial }\end{array}$ & $\begin{array}{c}\text { Plateformes de } \\
\text { covoiturage par } \\
\text { minibus }\end{array}$ & $\begin{array}{l}\text { Plateformes de } \\
\text { covoiturage } \\
\text { interurbain }\end{array}$ \\
\hline $\begin{array}{c}\text { Partenaires } \\
\text { essentiels }\end{array}$ & $\begin{array}{l}\text { Conducteurs } \\
\text { possédant leur } \\
\text { propre véhicule }\end{array}$ & $\begin{array}{c}\text { Fabricants automo- } \\
\text { biles célèbres } \\
\text { Conducteurs } \\
\text { professionnels }\end{array}$ & $\begin{array}{c}\text { Fabricants de } \\
\text { véhicules à énergies } \\
\text { nouvelles } \\
\text { Conducteurs } \\
\text { embauchés }\end{array}$ & $\begin{array}{l}\text { Conducteurs } \\
\text { possédant leur } \\
\text { propre véhicule }\end{array}$ \\
\hline $\begin{array}{l}\text { Proposition } \\
\text { de valeur }\end{array}$ & $\begin{array}{l}\text { Service pratique au } \\
\text { tarif plus bas que les } \\
\text { taxis normaux }\end{array}$ & $\begin{array}{l}\text { Service haut de } \\
\text { gamme de } \\
\text { véhicules luxueux }\end{array}$ & $\begin{array}{c}\text { Service de navettes } \\
\text { domicile-travail } \\
\text { confortables et } \\
\text { pratiques }\end{array}$ & $\begin{array}{l}\text { Service pratique et } \\
\text { flexible de } \\
\text { déplacements } \\
\text { intervilles }\end{array}$ \\
\hline $\begin{array}{l}\text { Segment de } \\
\text { clients }\end{array}$ & $\begin{array}{c}\text { Villes de } 1^{\mathrm{er}} \text { et } \\
2^{\mathrm{e}} \text { rangs/ } \\
\text { Axé principalement } \\
\text { sur les groupes de } \\
\text { consommateurs de } \\
\text { moyenne gamme et } \\
\text { bas de gamme }\end{array}$ & $\begin{array}{c}\text { Villes de } 1^{\mathrm{er}} \text { et } \\
2^{\mathrm{e}} \text { rangs/ } \\
\text { Clientèle d'affaires } \\
\text { haut de gamme }\end{array}$ & $\begin{array}{l}\text { Banlieue de grandes } \\
\text { villes de 1er rang/ } \\
\text { Utilisateurs vivant } \\
\text { en banlieue, mais } \\
\text { travaillant au } \\
\text { centre-ville }\end{array}$ & $\begin{array}{c}\text { Villes de } 3^{\mathrm{e}}, 4^{\mathrm{e}} \text { et } \\
5^{\mathrm{e}} \text { rangs } / \\
\text { Utilisateurs } \\
\text { nécessitant des } \\
\text { déplacements } \\
\text { interurbains }\end{array}$ \\
\hline $\begin{array}{l}\text { Structure des } \\
\text { coûts }\end{array}$ & $\begin{array}{l}\text { Investissement en } \\
\text { R-D des technologies/ } \\
\text { Publicité et autres } \\
\text { investissements } \\
\text { d'entreprise }\end{array}$ & $\begin{array}{l}\text { Coûts de mise en } \\
\text { place de la flotte et } \\
\text { embauche des } \\
\text { conducteurs }\end{array}$ & $\begin{array}{l}\text { Coûts de mise en } \\
\text { place de la flotte et } \\
\text { embauche des } \\
\text { conducteurs }\end{array}$ & $\begin{array}{l}\text { Coûts de gestion } \\
\qquad \text { du } \\
\text { fonctionnement } \\
\text { de la plateforme }\end{array}$ \\
\hline $\begin{array}{l}\text { Sources de } \\
\text { revenus }\end{array}$ & $\begin{array}{l}\text { Commissions des ser- } \\
\text { vices de covoiturage/ } \\
\text { Revenu de } \\
\text { coopération entre } \\
\text { entreprises }\end{array}$ & Frais de service & Frais de service & $\begin{array}{c}\text { Commissions des } \\
\text { services de } \\
\text { covoiturage }\end{array}$ \\
\hline
\end{tabular}

Tableau 1 - Comparaison des composantes de la MMA de Didi et des trois types de plateformes 
En raison d'une immense population et du niveau socioéconomique élevé, la plateforme de covoiturage en ligne est plus susceptible d'avoir un vaste impact dans les grandes villes. Il s'agit de la zone de marché où des entreprises de plateformes de covoiturage en ligne telles qu'Uber et Didi ont vu le jour et ont grandi. C'est également le secteur le plus compétitif du marché du covoiturage en ligne. La principale zone de marché de Didi est constituée des villes de premier et deuxième rangs, et sa proposition de valeur consiste à fournir des services de covoiturage à bas prix et pratiques aux consommateurs de masse. Après des années de concurrence féroce sur le marché, Didi a obtenu $90 \%$ des parts du marché et est devenue la seule véritable superpuissance du marché du covoiturage en Chine. Actuellement, elle se concentre sur les villes de premier et deuxième rangs, et s'étend progressivement à d'autres grandes villes à travers le monde pour gagner en importance.

À cause d'une constante concurrence du marché, des fusions et des acquisitions, un grand nombre de plateformes de covoiturage du même type que Didi ont dû se retirer du marché chinois puisqu'elles n'arrivaient pas à gagner en importance. Toutefois, certaines plateformes de petite et moyenne taille trouvent encore des occasions d'affaires et des possibilités de développement grâce à des études de marché détaillées et à des analyses. Au moyen de la segmentation des marchés, elles visent des groupes spécifiques d'utilisateurs et mettent de l'avant la proposition de valeur équivalente pour construire des modèles d'affaires ayant leurs propres caractéristiques.

\section{Partenaires essentiels}

Didi et les plateformes de covoiturage interurbain reposent principalement sur les véhicules privés, tandis que les plateformes haut de gamme et de covoiturage par minibus sont principalement soutenues par les fabricants automobiles.

\section{Proposition de valeur}

Tandis que Didi vise à fournir un service pratique à un tarif plus bas que les taxis normaux, les plateformes haut de gamme offrent des services haut de gamme de véhicules luxueux, les plateformes de covoiturage par minibus offrent des services de navettes domicile-travail confortables et pratiques, et les plateformes de covoiturage interurbain offrent des services de transport intervilles pratiques et flexibles.

\section{Segment de clients}

La zone de marché principale de Didi se situe dans les villes de premier et deuxième rangs. Elle se concentre sur les groupes de consommateurs de moyenne gamme et bas de gamme. Ce groupe d'utilisateurs est sensible à la tarification et la stratégie du faible prix aide la plateforme à prendre rapidement de l'ampleur. Au contraire, les plateformes haut de gamme visent la clientèle d'affaires haut de gamme dans les villes de premier et deuxième rangs. Ce type de groupe d'utilisateurs a un grand pouvoir d'achat et accorde plus d'attention aux services de grande qualité. Cette caractéristique de faible sensibilité à la tarification fait en sorte que la base d'utilisateurs d'HBP est plus stable que celle de Didi. Quant aux plateformes de covoiturage par minibus, leur principale zone de marché est les banlieues des grandes villes de premier rang. Leurs utilisateurs sont les voyageurs qui habitent en banlieue, mais qui travaillent au centre-ville. En comparaison avec Didi, elles possèdent une capacité de transport plus grande et plus stable pour les travailleurs à l'heure de pointe du matin et du soir. Enfin, les zones de marché cible pour les plateformes de covoiturage interurbain sont les villes de troisième, quatrième et cinquième rangs. Elles sont axées sur les utilisateurs ayant des besoins de déplacement interurbain.

\section{Structure des coûts et sources de revenus}

En raison des différentes sources d'approvisionnement en véhicules et en conducteurs de chacune des plateformes, la structure des coûts et leurs sources de revenus respectives sont également différentes (voir tableau 1).

Pour terminer, on peut constater que toutes les plateformes de petite et moyenne taille s'appuient sur leurs propres caractéristiques et avantages pour acquérir une part de marché en particulier. Elles cherchent une orientation de développement potentielle en établissant une proposition de valeur et un mode de fonctionnement différents de Didi, ce qui leur permet ainsi de gagner de l'espace de développement dans un marché presque monopolisé. 


\section{Conclusion}

Cet article visait à explorer comment les plateformes d'économie collaborative de petite et moyenne taille obtiennent un avantage concurrentiel et ont la chance de survivre et de se développer sur un marché monopolisé par des géants de l'industrie. Pour le marché du covoiturage en particulier, nous avons analysé 128 plateformes de petite et moyenne taille en Chine et avons résumé leurs trois principales catégories de plateformes: les plateformes haut de gamme, de covoiturage par minibus et de covoiturage interurbain En nous servant de la matrice de modèle d'affaires (MMA) comme outil d'analyse, nous avons comparé ces trois types de plateformes avec le géant de l'industrie Didi. Nous avons alors identifié certains des caractéristiques, initiatives et avantages concurrentiels de ces plateformes de covoiturage de petite et moyenne taille dans le contexte chinois.

Bien que Didi possède $90 \%$ des parts du marché et soit devenue la seule véritable superpuissance, des centaines de plateformes de petite et moyenne taille coexistent et trouvent des occasions d'affaires et des possibilités de développement. En comparaison avec Didi, les trois principaux types de plateformes présentent différentes caractéristiques dans leurs composantes (partenaires essentiels, proposition de valeur, segment de clients, structure des coûts et sources de revenus). Ainsi, ces plateformes s'appuient sur leurs propres caractéristiques et avantages pour se développer dans une niche de marché particulière.

Cette recherche présente une image qui reflète le stade de développement des PEC de petite et moyenne taille dans un marché précis monopolisé par un géant industriel. En plus de fournir quelques références pour le développement des plateformes d'économie collaborative de petite et moyenne taille dans d'autres régions et industries, elle jette également un regard nouveau sur la reconfiguration de l'échange marchand selon la perspective des entreprises de petite et moyenne taille.

Actuellement, les géants du marché dominent entièrement presque tous les secteurs de l'économie collaborative. Une compétition homogène dans le secteur du marché grand public est irréaliste pour les plateformes de petite et moyenne taille. Ainsi, elles devraient continuer de gagner en force dans cette féroce compétition, et s'appuyer sur leurs propres caractéristiques et avantages pour s'enraciner profondément dans des secteurs particuliers. Plus précisément, elles devraient constamment effectuer une segmentation détaillée du marché et une identification des utilisateurs plus sophistiquée, et devraient développer des avantages concurrentiels différenciés afin de gagner plus d'espace sur le marché.

\section{NOTES}

1 Didi Chuxing, précédemment nommée Didi Kuaidi, est une entreprise technologique chinoise proposant un service de véhicules de tourisme avec chauffeur sur application mobile.

\section{RÉFÉRENCES}

Amit, R. et Zott, C. (2015). Business Models. Dans J. D. Wright (dir.), International Encyclopedia of the Social \& Behavioral Sciences (2nd ed., p. 33-36). Amsterdam, Pays-Bas : Elsevier. https://doi.org/10.1016/B978-0-08-097086-8.73040-6

Analysys International. (2019). 2019 China ride-hailing market anabsis report. Beijing, Chine: Analysys International.

Baden-Fuller, C. et Mangematin, V. (2013). Business models: A challenging agenda. Strategic Organization, 11(4), 418-427. https://doi.org/10.1177/1476127013510112

Baidu. (2019). Congestion on the Beijing subway during the morning rush hour. Beijing, Chine: Baidu. Repéré à https:/ / baijiahao.baidu.com/s?id=1625807410247524627\&wfr=spider\&for=pc

Baijiahao. (2018). High-end and convenient ride-hailing platform "ReachNow" landed in China. Pékin, Chine: Beijing Zhiyue Network Technology Co., Ltd. Repéré à https://baijiahao.baidu.com/s?id=1619931556920448035\&wfr=spider\&for=pc

Bashir, M., Yousaf, A. et Verma, R. (2016). Disruptive business model innovation: How a tech firm is changing the traditional taxi service industry. Indian Journal of Marketing, 46(4), 49-59. https://doi.org/10.17010/ijom/2016/v46/i4/90530 
Beijing Bureau of Statistics. (2019). Beijing statistical yearbook 2019. Beijing, Chine: BBS.

Beijing Transportation Development Research Institute. (2019). Beïing traffic development annual report 2018. Beijing, Chine: BTDRI. Repéré à http://www.bjtrc.org.cn/List/index/cid/7.html

Berger, R. (2016). 2016 China tailored taxi service market analysis report. Shanghai, Chine: 199IT. Repéré à http:/ /www.199it.com/ archives/451262.html

Botsman, R. et Rogers, R. (2010). What's mine is yours: How collaborative consumption is changing the way we live. New York, NY : Penguin Press. https://doi.org/10.1016/S0168-9525(00)00086-X

China Industry Research Institute. (2018). Research report on the current situation and development prospect of China's online ride-bailing market in 2018. Shenzhen, Chine: CIRI.

China State Information Center. (2018). China annual report on sharing economy development 2018. Beijing, Chine: CSIC. Repéré à http://www.199it.com/archives/841592.html

China State Information Center. (2019). China annual report on sharing economy development 2019. Beijing, Chine: CSIC.

Clewlow, R. R. et Mishra, G. S. (2017). Disruptive transportation: The adoption, utilization, and impacts of ride-hailing in the United States. Berkeley, CA: Institute of Transportation Studies. Repéré à https://steps.ucdavis.edu/wp-content/uploads/2017/10/ ReginaClewlowDisuptive'Transportation.pdf

Cosenz, F. (2017). Supporting start-up business model design through system dynamics modelling. Management Decision, 55(1), 57-80. https://doi.org/10.1108/MD-06-2016-0395

Didi. (2016). 2015 big data report on China's intelligent mobility. Beijing, Chine: Didi.

Eckhardt, G. M. et Bardhi, F. (2015). The sharing economy isn't about sharing at all. Harvard Business Review, 28(1). Repéré à https://hbr.org/2015/01/the-sharing-economy-isnt-about-sharing-at-all

Edelman, B. G. et Geradin, D. (2015). Efficiencies and regulatory shortcuts: How should we regulate companies like Airbnb and Uber? Stanford Technology Law Review, 19, 293-328. https://dx.doi.org/10.2139/ssrn.2658603

Edelman, B., Luca, M. et Svirsky, D. (2017). Racial discrimination in the sharing economy: Evidence from a field experiment. American Economic Journal: Applied Economics, 9(2), 1-22. https://doi.org/10.1257/app.20160213

Ert, E., Fleischer, A. et Magen, N. (2016). Trust and reputation in the sharing economy: The role of personal photos in Airbnb. Tourism Management, 55(august 2016), 62-73. https://doi.org/10.1016/j.tourman.2016.01.013

Ertz, M., Hallegatte, D. et Bousquet, J. (2019). Retour vers le futur de l'échange marchand. Organisations \& territoires, 28(2), 99-113. https://doi.org/10.1522/revueot.v28n2.1052

Ertz, M. et Leblanc-Proulx, S. (2018). Sustainability in the collaborative economy: A bibliometric analysis reveals emerging interest. Journal of Cleaner Production, 196, 1073-1085. https://doi.org/10.1016/j.jclepro.2018.06.095

European Commission. (2016). A European agenda for the collaborative economy. Bruxelles, Belgique: European Commission. Repéré à https://ec.europa.eu/growth/content/european-agenda-collaborative-economy-1_fr

Finance. (2018). Didi is suspected of industry monopoly and has a market share of over 90\%. Pékin, Chine: Gucheng. Repéré à https:/ / finance.gucheng.com/201809/3532458.shtml

Fjeldstad, Ø. D. et Snow, C. C. (2018). Business models and organization design. Long Range Planning, 51(1), 32-39. https://doi.org/10.1016/j.lrp.2017.07.008

Frenken, K. et Schor, J. (2017). Putting the sharing economy into perspective. Environmental Innovation and Societal Transitions, 23, 3-10. https://doi.org/10.1016/j.eist.2017.01.003

Gabel, D. (2016). Uber and the persistence of market power. Journal of Economic Issues, 50(2), 527-534. https://doi.org/10.1080/00213624.2016.1179060

Hamari, J., Sjöklint, M. et Ukkonen, A. (2016). The sharing economy: Why people participate in collaborative consumption. Journal of the Association for Information Science and Technology, 67(9), 2047-2059. https://doi.org/10.1002/asi.23552

Hua, J. et Ray, K. (2018). Beyond the precariat: Race, gender, and labor in the taxi and Uber economy. Social Identities, 24(2), 271-289. https://doi.org/10.1080/13504630.2017.1321721 
Joyce, A. et Paquin, R. L. (2016). The triple layered business model canvas: A tool to design more sustainable business models. Journal of Cleaner Production, 135, 1474-1486. https://doi.org/10.1016/j.jclepro.2016.06.067

Kim, K., Baek, C. et Lee, J. D. (2018). Creative destruction of the sharing economy in action: The case of Uber. Transportation Research - Part A: Policy and Practice, 110, 118-127. https://doi.org/10.1016/j.tra.2018.01.014

Mikhalkina, T. et Cabantous, L. (2015). Business model innovation: How iconic business models emerge. Advances in Strategic Management, 33(1), 59-95. https://doi.org/10.1108/S0742-332220150000033024

National Bureau of Statistics of China (2019). China city statistics yearbook, 2018. Beijing, Chine: NBS.

Osterwalder, A. et Pigneur, Y. (2010). Business model generation: A bandbooke for visionaries, game changers, and challengers. Hoboken, NJ: Wiley.

Rainbow Bus. (2020). Introduction of Rainbow Bus. Pékin, Chine: Beijing Zhiyue Network Technology Co., Ltd. Repéré à http://www.rainbow-bus.cn

Shouqi. (2020). Introduction of Shouqi z̧huanche. Pékin, Chine: Zhuanche. Repéré à https://www.01zhuanche.com

Smith, A. (2016). Shared, collaborative and on demand: The new digital economy. Washington, DC: Pew Research Center.

Sundararajan, A. (2014). Peer-to-peer businesses and the sharing (collaborative) economy: Overview, economic effects and regulatory issues. Repéré à https://docs.house.gov/meetings/SM/SM00/20140115/101613/HHRG-113-SM00-20140115-SD003-U1.pdf

Täuscher, K. et Laudien, S. M. (2018). Understanding platform business models: A mixed methods study of marketplaces. European Management Journal, 36(3), 319-329. https://doi.org/10.1016/j.emj.2017.06.005

Teece, D. J. (2010). Business models, business strategy and innovation. Long Range Planning, 43(2-3), 172-194. https://doi.org/10.1016/j.lrp.2009.07.003 